\title{
Using personal service assistant for direct manipulation in smart space
}

\author{
J. Y. Chen ${ }^{1} \&$ T. Jeng ${ }^{2}$ \\ ${ }^{1}$ Department of Architecture, Nanya Institute of Technology, Taiwan \\ ${ }^{2}$ National Cheng-Kung University, Taiwan
}

\begin{abstract}
For embodying "Ubiquitous Computing" and "Context-awareness" into smart space design, an interactive model based on "Direct Manipulation" and a smart spatial interface mediated by PDA is demonstrated in this study to emphasize the user's initiative in actuating smart services in space. By the metaphor of WIMP (i.e. Window, Icon, Mouse and Popup-menu) inherited from computer desktop GUI, electronic services and digital information are bundled into physical objects in smart space. For implementation, versatile IT technology and spatial setting are integrated in laboratory, including the RFID, Wi-Fi 802.11 and IR network for context-sensing, device-controlling and service actuation. Through the evaluation in lab demo scenarios, the findings are as follows: (1) the cognitive mapping structure between digital and physical objects derives innovative research issues in user's spatial representation and object labelling for both individuals and groups; (2) the real-time mapping between spatial setting and user status abstracted from sensing network provides rich support for direct manipulation interactions for smart applications; (3) the PSA provides for the habitant extended ability of handheld control-and-display for almost everything. Keywords: ubiquitous computing, direct manipulation, smart spatial interface, object-aware, personal service assistant, RFID.
\end{abstract}

\section{Introduction}

Digital media are incrementally moving beyond digital design tools to interleaving themselves to be integral part of our living environments, especially in an interactive smart space. "Ubiquitous Computing", "Context-aware" and "Attentive Object" [5] provide new perspectives in developing digital 
architecture and smart living. Governmental ICT policies and related projects, entitled as "U-Japan", "U-Korea" and "U-Taiwan" [6], are declaring the next coming ubiquitous era for our everyday living. The smart living proposed in these projects are supported by environments embedded with sensing, communication and computing technology to make possible the integration of the two parallel worlds - digital and physical. However, information which is ubiquitous but overloaded via heterogeneous digital media has to be selectively presented in space at the right situation using the right interface (e.g. tabs, pads or boards). Context-aware services whether mediated by mobile or stationary devices and with active or passive smart applications, have to be provided intuitively to arouse habitants' interaction with the current context. Unconnected devices and multiplex control methods make our digital life filled with cognitive frictions. How to achieve interactive-awareness between habitants and smart space so as to support the subtlety of everyday life becomes our goal and challenge in this study. Based on the approach of "Direct Manipulation", a smart space with tangible interface, digital media, multiple display and wireless sensing network (e.g. RFID, IR and Wi-Fi) is experimented. In order to help the habitants navigate digital information and access chip-controlled spatial services in a common way, collaborative modules of PSA (Personal Service Assistant - a mobile device with abilities of RFID reading and IR controlling) and AIE (Ambient Intelligent Environment) are developed [7]. In this paper, the focus to be discussed is on the PSA applications in this project.
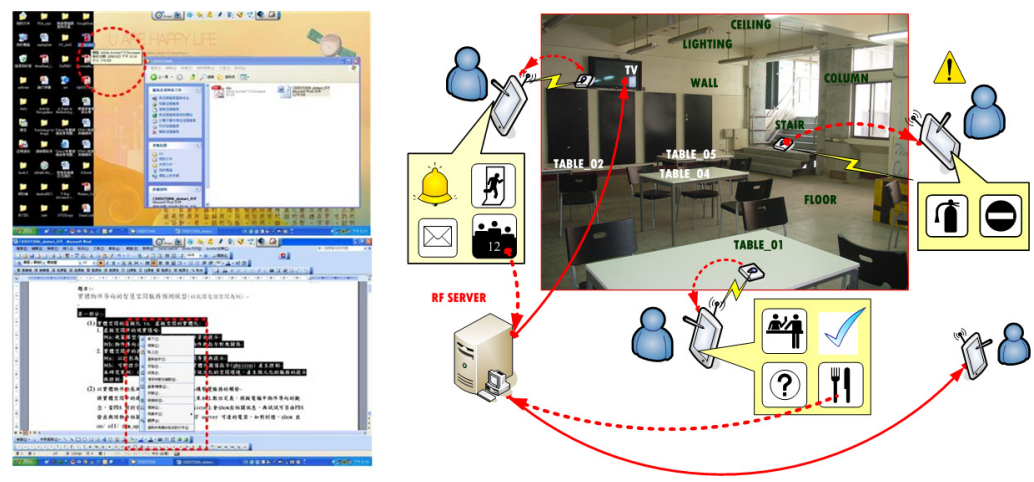

Figure 1: Direct manipulation on window desktop system for file annotation and selection sets menu (left); Direct manipulation proposed for smart spatial interface by PSA (right).

\section{Literature review}

Related studies apply the concept of semantic location model [8] in CAAD database to the prediction of user's next-coming location for triggering locationbased services. This literature depicts the location prediction by pattern learning and data-mining mechanism and simulated on two-dimensional CAAD platform 
prototype with fixed plan layouts. However, in the real world, the following problems are learned: (1) furniture, fixture or partitions are apt to move and should be able to report to the database on real-time basis; (2) user's nextcoming location predicted by statistical result of possibilities is unpredictable while the rates for each directions are close; (3) even located in the same point of a 2D plan, user's intention can be versatile in the three-dimensional space. Based on the above concern, instead of triggering services by location prediction, a diverse approach considered in this study is to support direct manipulation and trigger location-aware services by sensing network in real 3D space. Habitants are able to take the initiative in approaching to their attentive objects (fixed or movable) and intuitively trigger their intended object-dependent services. With the customized digital support from the PSA, the habitants interact with locationaware services on sensing-proximity basis.

\section{Previous study: Smart Spatial Interface}

People in this "Ubiquitous-era" need an interface system (or mediator) to coordinate the ubiquitous media and computing devices in space. GUI benefited from the metaphor of real-world desktop (e.g. folders, files, etc.) successfully provide hints to the user by mapping real-life experience to the computer desktop. It follows the essence of "Direct Manipulation" and WYSWYG (what you see is what you get); it is composed of window, icon, mouse, pull-down (or popup) menu and is operated by object-oriented command methods and mouse behaviors (ie. button clicking, rolling over, scrolling, etc). With the functions supported by object-oriented methods (e.g. pop-up message-box and service command menu), the GUI desktop displays for each virtual object its corresponding annotation and commands (e.g. icons, selection sets, etc.) which are not available yet for real objects in the physical world. The situated interaction provided under the framework of WIMP inspired this study in bundling electronic services and digital information into physical objects in smart space under the hierarchy of: (1) "Window", the proximity of user's locale anchored by RFID sensing network, (2) "Icons" (or "Phicons"), physical objects attached with RFID tags, (3) "Mouse", a PDA enhanced with RFID-reading and IR device control ability, and (4) "Popup-menus", prompts of object-aware service on a PDA screen (Figure 2).

In the lab works, the Smart Spatial Interface (SSI) proposed in this study is separated into two main developing directions: (1) PSA: user-centred contextaware mobile device and (2) AIE: infrastructure for supporting context-aware smart space by modules of "Event Awareness", "Service Actuation", and "Environmental Status". The PSA interacts with the AIE through user's direct manipulation on spatial physical element and simultaneously call the linkage to database on object basis. Object (i.e. physical element identified with RFID tag) then is selected as the context cue in triggering related spatial services. Most of the physical objects (e.g. photos, books, electronic appliances, door, chair, etc.) are embedded with digital meanings (e.g. content display, action control, etc.). The SSI helps habitant to freely navigate the digital information implicitly 
carried by components in space and provides situational control menu for currently activated objects. The PSA performs as the mouse in physical space for direct manipulation and the AIE provides supporting environment for service applications actuated by the PSA. The objectives for SSI are: (1) to achieve a universal user interface for smart space; (2) to achieve interactive awareness between user handheld devices (i.e. the PSA) and embedded intelligence in space (i.e. the AIE); (3) to provide real-time object-based annotation for navigation and menu for service control using mobile device; (4) to provide real-time monitoring for people and object status in space. The system model and AIE details are described in related publication [7].

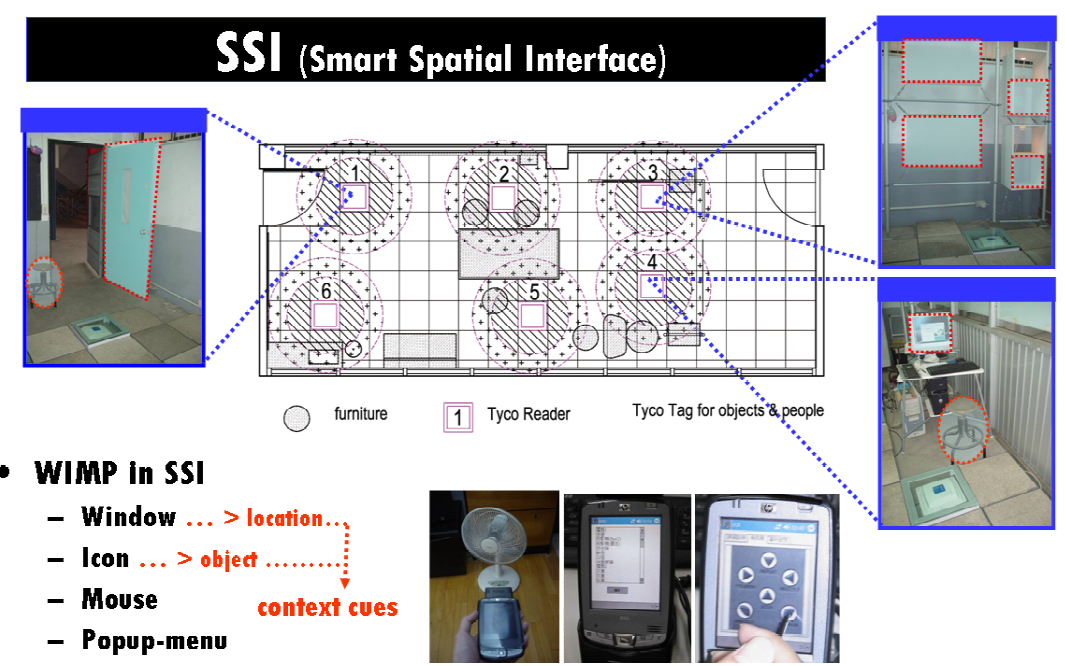

Figure 2: The Window, Icon, Mouse and Popup-menu represented in Smart Spatial Interface.

\section{Pointing Device in Smart Space: PSA}

A mobile device has become an extension of the person who holds it. These devices are in a perfect position to foster existing interactions and create opportunities for new human-space interactions. In this study, a PSA (Personal Service Assistant) is developed from the market-popular product of WinCEbased PDA with wireless network connecting ability and further enhanced with RFID CF reading ability to interact with any tagged objects in space (e.g. building element, furniture, appliances, personal belongings, etc.). The PSA applies the device-oriented location capability for user-oriented applications to preserve user's initiative. Except for the original functions supported by the PDA (e.g. touch-screen input, calendar, notebook, dictionary, calculator, address book, etc.), at current stage, the main applications appended to the PSA is focus on mediating user's (1) navigation in surrounding environment; (2) personal cross- 
product cross-species service control interface for any device and media in space. Whenever a person moving in space with the PSA, just like the mouse cursor moving over the icons in computer desktop, user accesses the hidden information of any attentive object and selects on screen from its corresponding service menu which is object-dependent and context-aware. The wireless control signal then is sent from the PSA to RF server or IR server and from server to devices for executing the chosen command. For demonstrating the above idea, sample applications which include scenarios of (1) Message Recall, (2) Media Play, (3) Device Control, and (4) Door Open are implemented (Figure 3).
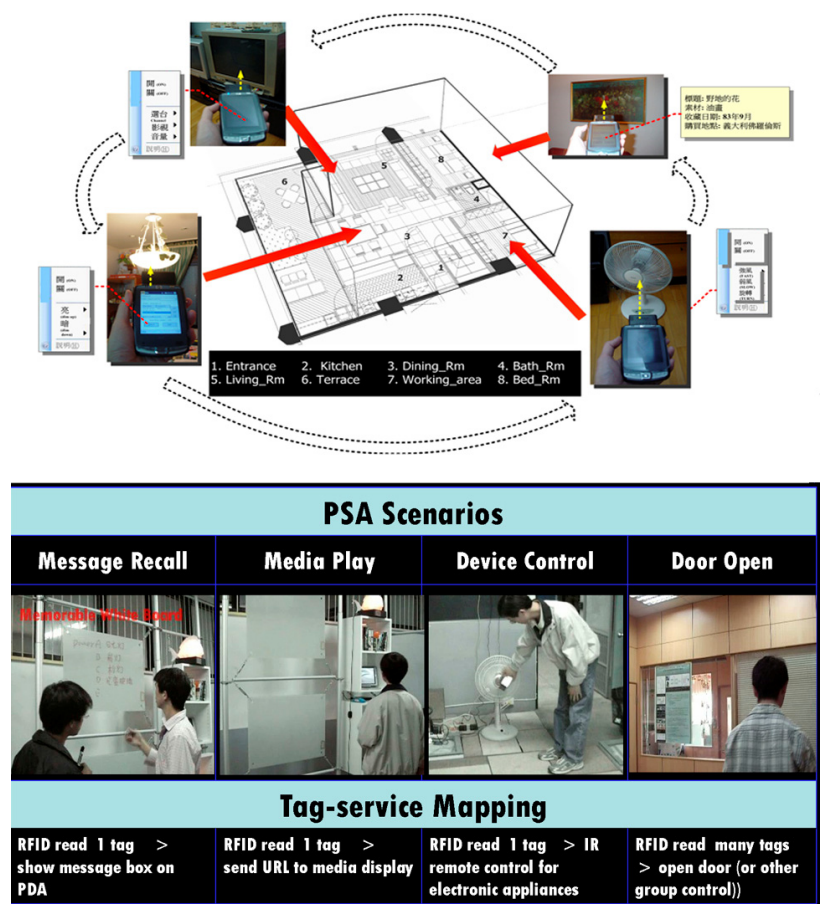

Figure 3: Conceptual diagram for Personal Service Assistant and related application scenarios, the demo videos are available at http://www.nanya.edu.tw/archi/SSI.htm.

\section{Object-oriented service providing and data representation}

Objects in space can be represented in different taxonomy, granularity requirement and working methods. The roles of objects in this study are: (1) target of direct manipulation, (2) physical-icons (or "Phicons") in space, (3) context cues for interactive awareness, (4) mediator between the PSA and AIE, and (5) portal for service actuation. The related study - "Attentive object" [5] also provides an object-based view in enriching people's natural interaction with 
everyday objects. Mobile phones become an on-the-move mediator for collecting object's just-in-time information from web search. Based on RFID, Bluetooth, Infra Red technology, user's attention is able to be sensed by object and become an input to interact with people, e.g. Moving Portrait. Comparing with the implicit input style by user's attention used in [5], in this study, a more precise and explicit input style is proposed by using the PSA. And, for output aims, in this study, unlimited to information and media display, a further objective on object-aware control is demonstrated. The object data will adaptively response to user's requirement by reasoning with user's current context which includes the short-term data, (i.e. current location, previously interacted object) and the longterm data, (i.e. personal preference).

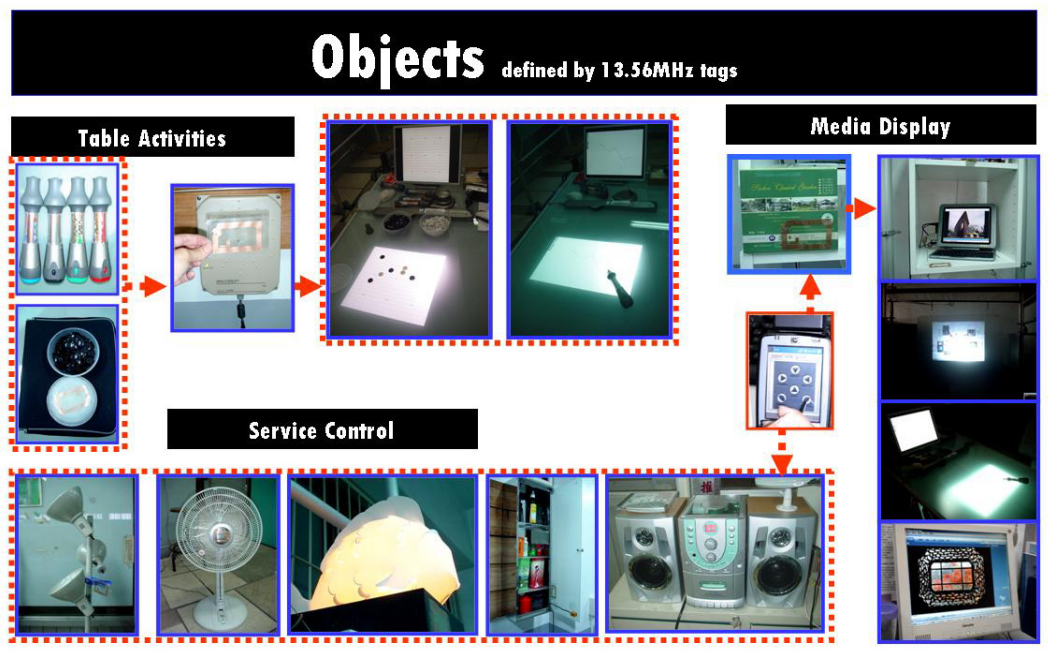

Figure 4: Object-aware service-providing (e.g. control, display and table works) in ambient intelligence environment.

In the digital world of the object-oriented information model, the term "object" represents not only real entities but also virtual ones, e.g. calendar, itinerary, syllabus, etc. In this study, a RFID-tagged-object stands for a pair of object which is physically and virtually connected to each other. For every single object (or group of objects), people may have diverse definitions according to their personal view and user goal. For example, a TV can be a media or videogame player and simultaneously a product for dealer. Based on previous spatial coding summary [7], and following the stages of object-oriented analysis (i.e. finding class-\&-object, identifying structures, identifying subjects, defining attributes, defining services), the tagged objects for SSI in this study are defined with class (e.g. TV, Lighting, A/C, etc.) and methods (e.g. Power_on, Voulmn_slider, etc.) (Figure 5). Following the characters of object-oriented data model, object are conceptually with: (1) attributes, operation methods, relations 
to other objects and identity, (2) class and instance, (3) inheritance from parent class, (4) compound and encapsulation, (5) part reuse, (6) "Noun-Verb" operation (7) object representation models for functional, static and dynamic. [9]

The tables in database are in three categories: (1) fundamental attributes for elements in space (e.g. TV, sofa, table, chair, people, tag and reader, and their background description); (2) definitions for tag-object relationship (one-to-one for normal cases, many-to-one for larger-size objects, one-to-many for objectgroup commands, which is used for decision- making strategy); (3) event records (stores every event and service actuation for safety and machine-learning use).

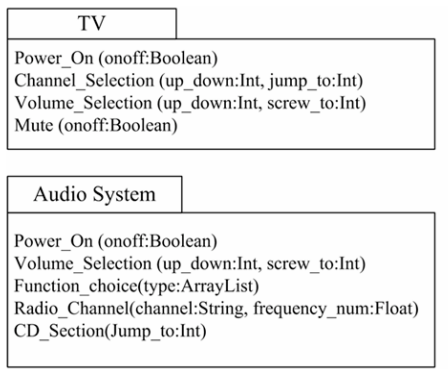

\begin{tabular}{|l|}
\hline \multicolumn{1}{|c|}{ Lighting System } \\
\hline $\begin{array}{l}\text { Light_choice(number:ArrayList) } \\
\text { Power_On (onoff:Boolean) } \\
\text { Bright_Selection (up_down:Int, adjust_to:Int) }\end{array}$ \\
\begin{tabular}{|l|}
\hline \multicolumn{1}{|c|}{ A/C System } \\
\hline $\begin{array}{l}\text { Power_On (onoff:Boolean) } \\
\text { Temperature_Selection (up_down:Int, adjust_to:Int) } \\
\text { Wind_Speed_Select (up_down:Int, adjust_to:ArrayList) } \\
\text { Rotation_Selection (Left_Right:ArrayList) }\end{array}$ \\
\hline
\end{tabular}
\end{tabular}

Figure 5: Object-oriented data model represented by class and methods, e.g. TV, Audio, Lighting, and A/C system.

\section{Implementation}

\subsection{System architecture}

The system is implemented on the basis of current Wi-Fi networked lab space and further installed with RFID sensing and Infra Red device control networks. The components consisted in the system architecture (Figure 6 - upper) are: (1) PSA: reads the tag id and passes to Smart Service Server via Wi-Fi network; receives signal from Smart Service Server and generates real-time message box or service page on screen; sends back user command to Smart Service Server for control actuation and become a cross-platform smart controller (Figure 6 lower); (2) RF Device Control Server: receives action commands from Smart Service Server via Wi-Fi network and sends actuation command to RF devices, e.g. lighting and power control, etc. or to IR devices, e.g. projectors, audio player, etc. (3) Database Server: system data centre for records of meaningful events and actions. (4) Smart Service Server: context-manager in coordinating the whole system.

\subsection{Technology}

The system integration developed in this study is focused on RF- and IR-based control for home appliance, (e.g. projector, lighting, audio etc.) and is coded by 
Java and eVB (Embedded Visual Basic) languages. The program module keeps listening to the values transmitted from the external world via RS-232 and sending them to both PC-08 RF Server and RP-08 IR Server (or PS-201) to achieve the purpose of electronic appliances control. The RPC (remote procedure call) supported by Wi-Fi technology enables the eVB programs on the PSA to request its call, via the wireless Wi-Fi network, from the VB, JAVA programs on server and to get response for application. Currently, socket for RPC is applied in this study and the standards of CORBA or Java RMI will be followed to support further interoperability.
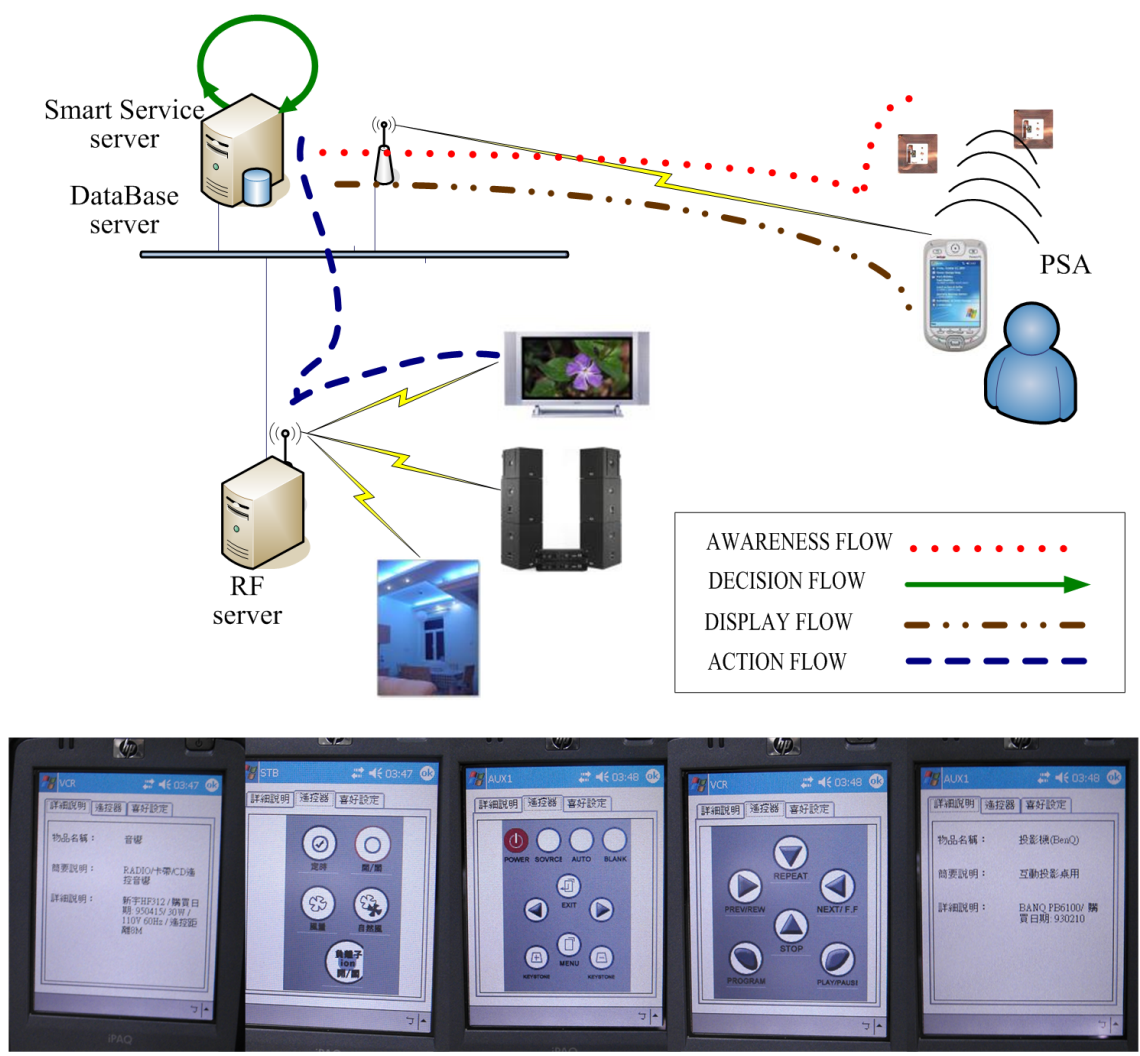

Figure 6: System architecture and information flows (upper) PSA screen pages for object annotation and service controls, e.g. fan, projector, audio player (lower).

In this study, RFID (Radio Frequency Identification) is the main technology used in connecting objects. A RFID system consists of reader, transponder (tag) and back-end API [10]. Species of RFID are identified by (1) Tag type: passive, semi-active or active, according to its power supply. (2) Frequency: $135 \mathrm{KHz}$, 
13.56 MHz, 860 930 MHz (UHF) and $2.45 \mathrm{GHz}$. In this study, two species of RFID system are applied, i.e. $13.56 \mathrm{~Hz}$ for the PSA and UHF for smart floor. (Table. 2) The major concern for mobile reader is the limited power supply by the PDA which greatly reduced the sensing proximity. And the major problem caused by the fixed UHF reader is how to adjust the tag into optimal status to keep the sensing proximity.

Table 1: Hardware specification.

\begin{tabular}{|c|c|c|c|}
\hline Name & Type & Specification & Brand \\
\hline \multirow{4}{*}{$\begin{array}{c}\text { Smart } \\
\text { Home } \\
\text { Devices }\end{array}$} & PC-08 RF Server for PC & Emit. Dist $>80 \mathrm{M}$ (non-blocked) & TECHCITY \\
\hline & RP-08 IR Server & $\begin{array}{l}\text { Receive Dist }>60 \mathrm{M} \text { (non-blocked) } \\
\text { Emit. Dist }>7 \mathrm{M} \text { (straight) }\end{array}$ & TECHCITY \\
\hline & $\begin{array}{l}\text { RC-08 3D Remote } \\
\text { Controller }\end{array}$ & $\begin{array}{l}\text { Work with Remote controller or } \\
\text { RF server }\end{array}$ & TECHCITY \\
\hline & $\begin{array}{l}\text { WS Lighting Switch } \\
\text { PS RF Switch }\end{array}$ & $\begin{array}{l}\text { RF wireless carrier wave for } \\
\text { remote control }\end{array}$ & TECHCITY \\
\hline PDA & HP iPAQ hx2410 & $\begin{array}{l}\text { Processor: Intel(R) PXA270 } \\
\text { OS: Windows CE } 4.21\end{array}$ & HP \\
\hline
\end{tabular}

Table 2: $\quad$ RFID system specification.

\begin{tabular}{|c|c|c|c|c|}
\hline Type & Product & Frequency & Brand & Distance \\
\hline Fixed Reader & $\begin{array}{c}\text { agile 2 reader with OmniWave } \\
\text { Antenna }\end{array}$ & UHF & Sensormatic & $\begin{array}{c}50 \mathrm{~cm} \\
\text { passive tag }\end{array}$ \\
\hline Mobile Reader & $\begin{array}{c}\text { V720S-HMF01 } \\
\text { For PDA CF type }\end{array}$ & $13.56 \mathrm{MHz}$ & Omron & $\begin{array}{c}10 \mathrm{~cm} \\
\text { passive tag }\end{array}$ \\
\hline
\end{tabular}

\section{Conclusion}

In this study, on the objective of developing a ubiquitous smart space, and with the approach of object-awareness, a smart spatial interface (SSI) with mobile device (PSA) is demonstrated to achieve direct manipulation. Findings from this study are: (1) For user applications - the PSA provides for the habitant extended ability of handheld control-and-display for almost everything. The cross-product object-aware interface reduces habitant's cognitive load in acquiring any digital service with personal preferences. Future potentials are providing modes for children, blind people, language translation and group control. (2) For objectmanagement - the object-oriented approach suggested in this study is very userintuitive in self-updating object information by simply initiating the labelling tags and loading product API via a well-designed server page and attaching/replacing to the new/old objects. (3) For further research issues conflict compromise mechanisms for multi-device and multi-person contexts and digital-physical mapping structure derived from spatial representation will be the next-step research issues for this study. 


\section{Acknowledgement}

Thanks to ROC National Science Council for Grant No. 94-2211-E-253-004.

\section{References}

[1] Weiser, M., The Computer for the 21st Century, Scientific American, Vol. 265, No. 3, pp. 66-75, 1991.

http://www.ubiq.com/hypertext/weiser/SciAmDraft3.html

[2] Schilit, B. \& Theimer, M. Disseminating Active Map Information to Mobile Hosts, IEEE Network 8(5), pp. 22-32, 1994.

[3] Dey, A K., Salber D., and Abowd, G D., A Context-based Infrastructure for Smart Environments, Proc. of the 1st International Workshop on Managing Interactions in Smart Environments (MANSE '99), Dublin, Ireland, pp. 114-128, 1999.

[4] Shneiderman, B., Direct manipulation: a step beyond programming languages, IEEE Computer 16(8), pp.57-69, August 1983.

[5] Maes, P., Attentive objects: enriching people's natural interaction with everyday objects, ACM Digital Library Interactions, Vol. 12, Number 4, pp. 45-48, 2005.

[6] Hopes high for 'u-Taiwan' project, Taipei Times, p.11, Apr. 6, 2006, http://www.taipeitimes.com/News/biz/archives/2006/04/06/2003301278

[7] Chen, J.Y., Yeh, M.K., Interactive Awareness: between PSA and AIE, Proc. of Symposium on Digital Life Technologies 2006, Tainan, Taiwan, June 2006.

[8] Lee, Y., Choi, J. and Lertakkhanakul J., Developing A User Location Prediction Model for Ubiquitous Computing, Proc. of CAADFutures, Vienna, Austria, 2005.

[9] Alshawi, M., Unerwood, J., Applying Object-oriented Analysis to the Integration of Design and Construction, Automation in Construction 5 (1996), pp. 105-121, 1996.

[10] Finkenzeller, K., RFID Handbook: Fundamentals and Applications in Contactless Smart Cards and Identification. John Wiley \& Sons, 2003. 